\title{
Maximum Contrast Beamformer for Electromagnetic Mapping of Brain Activity
}

\author{
Yong-Sheng Chen, Member, IEEE, Chih-Yu Cheng, Jen-Chuen Hsieh, and Li-Fen Chen*
}

\begin{abstract}
Beamforming technique can be applied to map the neuronal activities from magnetoencephalographic/electroencephalographic (MEG/EEG) recordings. One of the major difficulties of the scalar-type MEG/EEG beamformer is the determination of accurate dipole orientation, which is essential to an effective spatial filter. This paper presents a new beamforming technique which exploits a maximum contrast criterion to maximize the ratio of the neuronal activity estimated in a specified active state to the activity estimated in a control state. This criterion leads to a closed-form solution of the dipole orientation. Experiments with simulation, phantom, and finger-lifting data clearly demonstrate the effectiveness, efficiency, and accuracy of the proposed method.
\end{abstract}

Index Terms-EEG, electromagnetic brain mapping, maximum contrast beamformer, MEG.

\section{INTRODUCTION}

$\mathbf{M}$ AGNETOENCEPHALOGRAPHY (MEG) and electroencephalography (EEG) are tools for functional brain imaging that noninvasively measure the magnetic induction and scalp potentials, respectively, produced by the electrical brain activities. Compared to functional magnetic resonance imaging (fMRI) that detects the relatively slow hemodynamic changes which are the correlates of neuronal activities, MEG and EEG directly measure the neuronal activities with superior temporal resolution. This advantage enables MEG/EEG to offer the possibility to penetrate the brain dynamics and neuronal coupling between cell assemblies.

The electromagnetic field recorded by MEG sensors or EEG electrodes is the ensemble of neuronal activities within the whole brain. From the given primary current sources, how the external electromagnetic field should appear can be calculated

Manuscript received August 1, 2005; revised March 1, 2006. This work was supported in part by the National Science Council, Taiwan, under Grant NSC932213-E-010-006 and Grant NSC93-2218-E-009-025, in part by the Taipei Veterans General Hospital under Grant VGH94-364-1, and in part by the National Health Research Institutes under Grant NHRI-EX94-CD9401. Asterisk indicates corresponding author.

Y.-S. Chen and C.-Y. Cheng are with the Department of Computer Science, National Chiao Tung University, Hsinchu 300, Taiwan (e-mail: yschen@cs.nctu.edu.tw; chengcy@csie.nctu.edu.tw).

J.-C. Hsieh is with the Laboratory of Integrated Brain Research, Department of Medical Research and Education, Taipei Veterans General Hospital, Taipei 112, Taiwan and also with the Faculty of Medicine, the Institute of Neuroscience, National Yang-Ming University, Taipei 112, Taiwan (e-mail: jchsieh@vghtpe.gov.tw).

*L.-F. Chen is with the Center for Neuroscience, National Yang-Ming University, Taipei 112, Taiwan and also with the Laboratory of Integrated Brain Research, Department of Medical Research and Education, Taipei Veterans General Hospital, Taipei 112, Taiwan (e-mail: lfchen@ym.edu.tw).

Color versions of Figs. 1, 3, and 5 are available online at http://ieeexplore. ieee.org.

Digital Object Identifier 10.1109/TBME.2006.878115 according to the forward solutions [1], [2]. The electromagnetic inverse problem is to estimate the neuronal activities in the brain based on the MEG/EEG recordings [3], [4]. This kind of inverse problem is inherently ill-posed. Approximations such as the equivalent current dipole (ECD) model, assumptions such as a fixed number of dipoles within an epoch, and constraints such as the anatomical constraint and the minimum-norm constraint are usually required to obtain reasonable solutions for the inverse problem.

Dipole fitting is the most widely-used method for solving the inverse problem. This method assumes that the sources of brain activity consist of a fixed number of ECDs and estimates the parameters of the ECDs, including location, orientation, and strength, by minimizing the squared difference between the MEG/EEG recordings and the electromagnetic signals predicted by these ECDs [1], [3]. The major difficulty of the dipole fitting method is how to determine the a priori number of sources. Moreover, the involved minimization process may trap in local minima and result in significant localization errors if nonlinear simplex or gradient-based search is engaged [5]. As alternatives, MUSIC and its extensions [6]-[8] can avoid this problem by scanning through the region of interest and determining the locations with peak projections of forward models in the signal subspace. Another kind of inverse methods estimate the brain activities distributed on the cortical surface, which can be extracted from the magnetic resonance imaging (MRI) of the head. Each tessellation element of the surface model is associated with a current dipole, whose orientation is either set to be on the tangential plane or normal to the local cortical surface. This anatomical constraint leads to a linear estimation of dipole strengths distributed on the cortical surface. However, this estimation problem is usually underdetermined and regularization such as the minimum-norm constraint is required to obtain a unique solution [9]-[11]. This solution, unfortunately, tends to emphasize the cortical regions closer to the MEG sensors or EEG electrodes due to the preference of smaller dipole strength [12].

During the past decade, beamforming methods [13], [14], which are spatial filtering techniques that linearly integrate information over multiple spatially distributed sensors, have becoming more and more attractive for the localization of brain activities [15]-[20]. Beyond the topographic mapping of signal power at the sensors, we can obtain the tomographic mapping of source power within the head by using beamforming methods to scan the head region and reveal locations having significant neuronal activation. For inter-subject investigation of brain function, grand-average activation maps or group comparison results can be obtained from the individual tomographic mappings, after proper statistical flattening [12], [21]. 
MEG/EEG beamformer intends to concentrate the array of MEG sensors or EEG electrodes on the neuronal activities coming from only one particular position at a time. More specifically, this beamformer can obtain the activation magnitude of the targeted source by imposing the unit-gain constraint while suppressing the contribution from other sources by applying the minimum variance criterion. Given a unit dipole with specified position and orientation, this method can analytically calculate a spatial filter from the data covariance matrix and the lead field of this dipole. Filter output at this position can be obtained by passing the electromagnetic recordings through the calculated spatial filter. Individually repeating the above procedure for each position while scanning through the head region results in a distribution map of brain activity [15], [16].

There are two types of MEG/EEG beamformers in the literature. The first one is the vector-type beamformer [15], [17]. This approach decomposes the dipole into three orthogonal components, each one with a fixed orientation. Three spatial filters are analytically computed for these three orthogonal components and then can be used to estimate the output source power as well as the activity index in the linearly constrained minimum variance (LCMV) beamformer method [15]. Another type of MEG/EEG beamformers is the scalar-type beamformer [16], [22], in which only one spatial filter is used to estimate the brain activity for each targeted position. The dipole orientation is involved in the calculation of the spatial filter to maximize the output pseudo-Z statistic [16].

One of the advantages of the vector beamformer is that it is efficient to compute the spatial filters because all the involved procedures are deterministic. Compared to the vector beamformers, the scalar beamformer benefits from its higher output signal-to-noise ratio (SNR) and more focal spatial extent of the estimated brain activity distribution [22], [23]. Notice that it is essential to accurately determine the dipole orientation in the scalar-type beamforming methods. Only when the dipole orientation is accurate can result in effective spatial filter [23], [24]. If the dipole orientation is deviated from the ground truth, the spatial filter with high specificity may suppress the contribution from the true source and fail to reveal the source energy (see Section IV-A for more details).

One way to determine the dipole orientation is to simply align it to the local cortical surface normal [24], [25]. Unfortunately, surface reconstruction for convoluted cortex is very difficult and the reconstruction deviation will decrease the accuracy of the dipole orientation. Hillebrand and Barnes reported in [24] that the anatomical constraints can be advantageous only when the estimation error of the surface normal is smaller than $10^{\circ}$. In [16], Robinson and Vrba proposed the synthetic aperture magnetometry (SAM) method in which the dipole orientation was determined by maximizing the pseudo- $Z$ statistic. In general, it is computationally infeasible to obtain the optimal orientation by exhaustively evaluating all the possible candidates. Nonlinear optimization method is more efficient, but only can guarantee to find the suboptimal solution. Recently, Sekihara et al. proposed an optimal solution to the determination of dipole orientation that maximizes the output SNR (pseudo-Z statistic) [22]. The dipole orientation can be calculated very efficiently in a closed-form manner.
In this paper, we develop a novel spatial filtering technique, called the maximum contrast beamformer (MCB), for statistical mapping of neuronal activities. This MCB method has the advantages of good output SNR and focal activity distribution as in scalar beamformers, while the dipole orientation is determined accurately and efficiently. In addition to the unit-gain constraint and the minimum-variance criterion, as in the conventional beamformers, our method exploits a maximum-contrast criterion that maximizes the ratio of the reconstructed neuronal activities in the active state to those in the control state. The maximum-contrast criterion helps to analytically and accurately determine the dipole orientation in a closed-form manner. The spatial filter can, thus, be obtained very efficiently for each targeted position. Once the activity waveform is reconstructed in the source space by spatially filtering the electromagnetic recordings, an F-statistic map can be calculated to reveal cortical regions with significant difference of activities between the active and control states. Compared to the pseudo-Z statistic [16], [22] in which the sensor noise is considered, F statistic gives the statistical inference between two contrast states [12]. According to our experiments with simulation and phantom data, the MCB can estimate the dipole orientation and then locate the source, efficiently and accurately. When applied to a finger-lifting study, the F-statistic map computed from the movement-evoked field clearly identifies the sensorimotor area with high contrast. In this paper, we apply the MCB method for MEG studies. The same method can also be applied for EEG source localization.

\section{METHODS}

\section{A. Scalar Beamformer}

Consider a unit dipole with parameters $\theta=\left\{\mathbf{r}_{\mathbf{q}}, \mathbf{q}\right\}$, where $\mathbf{r}_{\mathbf{q}}$ is the dipole location and $\mathbf{q}$ is a unit vector representing the dipole orientation. Denote the $N \times 1$ column vector $\mathbf{l}_{\theta}$ to be the lead field vector of this unit dipole. The lead field vector $l_{\theta}$ contains the predicted measurements of $N$ MEG sensors that can be calculated by

$$
\mathbf{l}_{\theta}=\mathbf{L}_{\mathbf{r}_{\mathbf{q}}} \mathbf{q} \text {. }
$$

Here, $\mathbf{L}_{\mathbf{r}_{\mathbf{q}}}$ is the $N \times 3$ lead field matrix and can be derived from the forward solution [2], [4]. Now suppose the source strength of this dipole is $s_{\theta}(t)$. Let us decompose the MEG recordings $\mathbf{m}(t)$ into two components

$$
\mathbf{m}(t)=\mathbf{m}_{\theta}(t)+\mathbf{m}_{n}(t)
$$

where $\mathbf{m}_{\theta}(t)=s_{\theta}(t) \mathbf{l}_{\theta}$ denotes the predicted magnetic field originated from the targeted source with parameters $\theta$ and $\mathbf{m}_{n}(t)$ denotes the sensor noise plus the magnetic field originated from all other sources.

For the dipole source with parameters $\theta$, the ultimate goal of a scalar MEG beamformer is to determine a spatial filter $\mathbf{w}_{\theta}$, an $N \times 1$ column vector, such that the output signal $y(t)$ obtained by passing the MEG recordings $\mathbf{m}(t)$ through the spatial filter $\mathbf{w}_{\theta}$

$$
y(t)=\mathbf{w}_{\theta}^{T} \mathbf{m}(t)
$$


approximates the source strength $s_{\theta}(t)$ of this dipole. Toward this goal, the spatial filter can be determined by applying the unit-gain constraint, $\mathbf{w}_{\theta}^{T} \mathbf{l}_{\theta}=1$, and by minimizing the variance of the filter output $y(t)$ [15]. Because

$$
\begin{aligned}
y(t) & =\mathbf{w}_{\theta}^{T} \mathbf{m}(t) \\
& =\mathbf{w}_{\theta}^{T} \mathbf{m}_{\theta}(t)+\mathbf{w}_{\theta}^{T} \mathbf{m}_{n}(t) \\
& =s_{\theta}(t) \mathbf{w}_{\theta}^{T} \mathbf{l}_{\theta}+\mathbf{w}_{\theta}^{T} \mathbf{m}_{n}(t) \\
& =s_{\theta}(t)+\mathbf{w}_{\theta}^{T} \mathbf{m}_{n}(t)
\end{aligned}
$$

minimization of the variance of $y(t)$ means the suppression of the leakage, $\mathbf{w}_{\theta}^{T} \mathbf{m}_{n}(t)$, contributed from all other sources and sensor noise, while preserving the magnitude of the source strength $s_{\theta}(t)$. Therefore, the optimal spatial filter $\hat{\mathbf{w}}_{\theta}$ can be obtained by

$$
\begin{array}{r}
\hat{\mathbf{w}}_{\theta}=\arg \min _{\mathbf{w}_{\theta}}\left[E\left\{\|y(t)-E\{y(t)\}\|^{2}\right\}+\alpha\left\|\mathbf{w}_{\theta}\right\|^{2}\right] \\
\text { subject to } \mathbf{w}_{\theta}^{T} \mathbf{l}_{\theta}=1
\end{array}
$$

where $E\{\cdot\}$ denotes the expectation value and $\alpha$ is the parameter of Tikhonov regularization [26] for restricting the norm of the spatial filter $\mathbf{w}_{\theta}$. By substituting (3) into the above equation and solving the constrained optimization problem via the method of Lagrange multipliers, we can obtain the analytical solution of $\mathbf{w}_{\theta}$ [15], [16], [27]:

$$
\begin{aligned}
\hat{\mathbf{w}}_{\theta} & =\arg \min _{\mathbf{w} \theta} \mathbf{w}_{\theta}^{T}(\mathbf{C}+\alpha \mathbf{I}) \mathbf{w}_{\theta} \quad \text { subject to } \mathbf{w}_{\theta}^{T} \mathbf{l}_{\theta}=1 \\
& =\frac{(\mathbf{C}+\alpha \mathbf{I})^{-1} \mathbf{l}_{\theta}}{\mathbf{l}_{\theta}^{T}(\mathbf{C}+\alpha \mathbf{I})^{-1} \mathbf{l}_{\theta}}
\end{aligned}
$$

where $\mathbf{C}=E\left\{\|\mathbf{m}(t)-E\{\mathbf{m}(t)\}\|^{2}\right\}$ is the $N \times N$ covariance matrix of the MEG measurements $\mathbf{m}(t)$ and $\mathbf{I}$ is the $N \times N$ identify matrix.

\section{B. Statistical Mapping}

For each targeted position $\mathbf{r}_{\mathbf{q}}$, the spatial filter for the dipole with specified orientation $\mathbf{q}$ can be calculated by using (6). Once obtained, the spatial filter estimates the dipole activity at the targeted position $\mathbf{r}_{\mathbf{q}}$ by using (3). By scanning the head region and performing the above-mentioned beamforming procedure for each probed position individually, we obtain the activities of the whole head. Notice that the norm of the spatial filter is location-dependent. Compared to a superficial dipole, the lead field norm of a deeper dipole is smaller [1] and, thus, its corresponding spatial filter has a larger norm, as well as a larger response, according to the unit-gain constraint. There may be strong non-task-related activity in the filtered outputs. Therefore, the strength of the estimated activity is not necessarily proportional to the observability of a task-related source. We need a metric that can normalize task-related output by non-task-related output.

Beamforming methods provide statistical maps to reveal the regions having significant neuronal activities [15], [16]. Instead of the source power, we calculate the F statistic which is the variance ratio of the filtered activity estimated in an active state to that estimated in a control state. For each dipole source with parameters $\theta$, we calculate the spatial filter $\mathbf{w}_{\theta}$ by using (6) and then calculate the F statistic as

$$
\begin{aligned}
F_{\theta} & =\frac{E\left\{\left\|\mathbf{w}_{\theta}^{T} \mathbf{m}_{a}(t)-E\left\{\mathbf{w}_{\theta}^{T} \mathbf{m}_{a}(t)\right\}\right\|^{2}\right\}}{E\left\{\left\|\mathbf{w}_{\theta}^{T} \mathbf{m}_{c}(t)-E\left\{\mathbf{w}_{\theta}^{T} \mathbf{m}_{c}(t)\right\}\right\|^{2}\right\}} \\
& =\frac{\mathbf{w}_{\theta}^{T} \mathbf{C}_{a} \mathbf{w}_{\theta}}{\mathbf{w}_{\theta}^{T} \mathbf{C}_{c} \mathbf{w}_{\theta}}
\end{aligned}
$$

where $\mathbf{C}_{a}$ and $\mathbf{C}_{c}$ are the covariance matrices estimated from the MEG measurements in active and control states, $\mathbf{m}_{a}(t)$ and $\mathbf{m}_{c}(t)$, respectively. Therefore, the value of $F_{\theta}$ indicates the significant level that the neuronal activity is stronger in the active state than that in the control state at the targeted position $\mathbf{r}_{\mathbf{q}}$ with dipole orientation $\mathbf{q}$.

There are three covariance matrices involved in the beamforming process so far, that is, $\mathbf{C}, \mathbf{C}_{a}$, and $\mathbf{C}_{c}$. The matrix $\mathbf{C}$ is used to calculate the spatial filter and the corresponding time interval of $\mathbf{m}(t)$ should be large enough to contain meaningful activities. The matrix $\mathbf{C}_{a}$ is used to calculate the F-statistic value within the time interval of $\mathbf{m}_{a}(t)$. There are many options to estimate the covariance matrix $\mathbf{C}_{c}$ in the denominator of (7). For the dual-state MEG experiments, $\mathbf{C}_{c}$ can be calculated from the MEG recordings within the time window of the control state. In this case, the F-statistic map represents the contrast of the neuronal activation of the brain between the active and control states. The other way to calculate the covariance matrix $\mathbf{C}_{c}$ is to exploit the empty room MEG signals that can be recorded for a period of time when there is no subject in the shielding room. From these signals we calculate $\mathbf{C}_{c}$ and keep only the diagonal part while setting all other elements of this covariance matrix to be zero. The diagonal part of this covariance matrix can be regarded as the sensor gains without considering the correlations among different sensors. The F-statistic map in this case reveals regions having significant brain activity for the single-state MEG experiments. In another way, we can simply set $\mathbf{C}_{c}$ to be the identity matrix. This means that the sensors are assumed to have uniform gain and the sensor noises are independent and identically distributed.

\section{Maximum Contrast Beamformer}

The analytical solution of the spatial filter and the following F-statistic calculation are derived for a dipole with given parameters $\theta=\left\{\mathbf{r}_{\mathbf{q}}, \mathbf{q}\right\}$. The position parameters $\mathbf{r}_{\mathbf{q}}$ can be set to be the sampling positions sequentially. However, the dipole orientation $\mathbf{q}$ is difficult to determine.

Instead of the time-consuming exhaustive search or the suboptimal nonlinear search, we propose a closed-form solution to the determination of dipole orientation in the following. By substituting (1) into (6), we rewrite the solution of $\mathbf{w}_{\theta}$ as

$$
\begin{aligned}
\hat{\mathbf{w}}_{\theta} & =\frac{(\mathbf{C}+\alpha \mathbf{I})^{-1} \mathbf{L}_{\mathbf{r}_{\mathbf{q}} \mathbf{q}}}{\mathbf{q}^{T} \mathbf{L}_{\mathbf{r}_{\mathbf{q}}}^{T}(\mathbf{C}+\alpha \mathbf{I})^{-1} \mathbf{L}_{\mathbf{r}_{\mathbf{q}}} \mathbf{q}} \\
& =\frac{\mathbf{A}_{\mathbf{r}_{\mathbf{q}}} \mathbf{q}}{\mathbf{q}^{T} \mathbf{B}_{\mathbf{r}_{\mathbf{q}}} \mathbf{q}}
\end{aligned}
$$

where both $\mathbf{A}_{\mathbf{r}_{\mathbf{q}}}=(\mathbf{C}+\alpha \mathbf{I})^{-1} \mathbf{L}_{\mathbf{r}_{\mathbf{q}}}$ and $\mathbf{B}_{\mathbf{r}_{\mathbf{q}}}=\mathbf{L}_{\mathbf{r}_{\mathbf{q}}}^{T} \mathbf{A}_{\mathbf{r}_{q}}$ depend only on the dipole position $\mathbf{r}_{\mathbf{q}}$. We determine the optimal dipole orientation $\hat{\mathbf{q}}$ as the one that can maximize the contrast of the source power estimated in the active state to that estimated in 
the control state. By substituting (8) into (7) and maximizing the F statistic, we obtain

$$
\begin{aligned}
\hat{\mathbf{q}} & =\arg \max _{\mathbf{q}} \frac{\left(\frac{\mathbf{A}_{\mathbf{r}_{\mathbf{q}} \mathbf{q}}}{\mathbf{q}^{T} \mathbf{B}_{\mathbf{r}_{\mathbf{q}} \mathbf{q}}}\right)^{T} \mathbf{C}_{a}\left(\frac{\mathbf{A}_{\mathbf{r}_{\mathbf{q}} \mathbf{q}}}{\mathbf{q}^{T} \mathbf{B}_{\mathbf{r}_{\mathbf{q}} \mathbf{q}}}\right)}{\left(\frac{\mathbf{A}_{\mathbf{r}_{\mathbf{q}} \mathbf{q}}}{\mathbf{q}^{T} \mathbf{B}_{\mathbf{r}_{\mathbf{q}} \mathbf{q}}}\right)^{T} \mathbf{C}_{c}\left(\frac{\mathbf{A}_{\mathbf{r}_{\mathbf{q}}} \mathbf{q}}{\mathbf{q}^{T} \mathbf{B}_{\mathbf{r}_{\mathbf{q}} \mathbf{q}}}\right)} \\
& =\arg \max _{\mathbf{q}} \frac{\mathbf{q}^{T} \mathbf{A}_{\mathbf{r}_{\mathbf{q}}} \mathbf{C}_{a} \mathbf{A}_{\mathbf{r}_{\mathbf{q}}} \mathbf{q}}{\mathbf{q}^{T} \mathbf{A}_{\mathbf{r}_{\mathbf{q}}}^{T} \mathbf{C}_{c} \mathbf{A}_{\mathbf{r}_{\mathbf{q}} \mathbf{q}}} \\
& =\arg \max _{\mathbf{q}} \frac{\mathbf{q}^{T} \mathbf{P}_{\mathbf{r}_{\mathbf{q}}} \mathbf{q}}{\mathbf{q}^{T} \mathbf{Q}_{\mathbf{r}_{\mathbf{q}}} \mathbf{q}}
\end{aligned}
$$

in which the term $\mathbf{q}^{T} \mathbf{B}_{\mathbf{r}_{\mathbf{q}}} \mathbf{q}$ in both the numerator and denominator is a scalar and can be eliminated, the $3 \times 3$ matrix $\mathbf{P}_{\mathbf{r}_{\mathbf{q}}}=$ $\mathbf{A}_{\mathbf{r}_{\mathbf{q}}}^{T} \mathbf{C}_{a} \mathbf{A}_{\mathbf{r}_{\mathbf{q}}}$, and the $3 \times 3$ matrix $\mathbf{Q}_{\mathbf{r}_{\mathbf{q}}}=\mathbf{A}_{\mathbf{r}_{\mathbf{q}}}^{T} \mathbf{C}_{c} \mathbf{A}_{\mathbf{r}_{\mathbf{q}}}$. The solution of $\hat{\mathbf{q}}$ in the above equation is the eigenvector corresponding to the maximum eigenvalue of the matrix $\mathbf{Q}_{\mathbf{r}_{q}}^{-1} \mathbf{P}_{\mathbf{r}_{\mathbf{q}}}$. Because these two matrices, $\mathbf{Q}_{\mathbf{r}_{\mathbf{q}}}$ and $\mathbf{Q}_{\mathbf{r}_{\mathbf{q}}}^{-1} \mathbf{P}_{\mathbf{r}_{\mathbf{q}}}$, are both $3 \times 3$, we can solve the matrix inverse problem and the eigenproblem in a closed-form manner [28], [29]. In practice, we replace the matrix $\mathbf{Q}_{\mathbf{r}_{\mathbf{q}}}$ with the matrix $\mathbf{Q}_{\mathbf{r}_{\mathbf{q}}}+\beta \mathbf{I}$ to avoid the singularity problem, where $\beta$ is another regularization parameter and $\mathbf{I}$ is the $3 \times 3$ identity matrix.

Although there is an $N \times N$ matrix inverse process in the calculation of $\mathbf{P}_{\mathbf{r}_{\mathbf{q}}}$ and $\mathbf{Q}_{\mathbf{r}_{\mathbf{q}}}$

$$
\begin{aligned}
& \mathbf{P}_{\mathbf{r}_{\mathbf{q}}}=\mathbf{L}_{\mathbf{r}_{\mathbf{q}}}^{T}\left((\mathbf{C}+\alpha \mathbf{I})^{-1}\right)^{T} \mathbf{C}_{a}(\mathbf{C}+\alpha \mathbf{I})^{-1} \mathbf{L}_{\mathbf{r}_{\mathbf{q}}} \\
& \mathbf{Q}_{\mathbf{r}_{\mathbf{q}}}=\mathbf{L}_{\mathbf{r}_{\mathbf{q}}}^{T}\left((\mathbf{C}+\alpha \mathbf{I})^{-1}\right)^{T} \mathbf{C}_{c}(\mathbf{C}+\alpha \mathbf{I})^{-1} \mathbf{L}_{\mathbf{r}_{\mathbf{q}}}
\end{aligned}
$$

these two terms $\left((\mathbf{C}+\alpha \mathbf{I})^{-1}\right)^{T} \mathbf{C}_{a}(\mathbf{C}+\alpha \mathbf{I})^{-1}$ and $\left((\mathbf{C}+\alpha \mathbf{I})^{-1}\right)^{T} \mathbf{C}_{c}(\mathbf{C}+\alpha \mathbf{I})^{-1}$ are location-independent and can be calculated when both the MEG recordings are available and the time windows are set. Once calculated, these two terms can be used to derive $\mathbf{P}_{\mathbf{r}_{\mathbf{q}}}$ and $\mathbf{Q}_{\mathbf{r}_{\mathbf{q}}}$, the optimal dipole orientation $\hat{\mathbf{q}}$, and the spatial filter $\hat{\mathbf{w}}_{\theta}$ for each position $\mathbf{r}_{\mathbf{q}}$.

\section{EXPERIMENTS}

In this paper, we performed experiments, including simulations, phantom studies, and a finger movement study, to evaluate the accuracy of source localization and dipole orientation estimation by using the proposed MCB method. The magnetic signals were recorded from or simulated according to the 204 planar gradiometers of a whole-head neuromagnetometer (Vectorview system, Neuromag Ltd., Finland). The homogeneous spherical model was adopted as the head conductor model in the calculation of forward solutions.

\section{A. Simulations}

Three dipole sources with temporal profiles of sinusoidal waves were located in the brain, as shown in Fig. 1(a) and (b). Notice that the structural MRI shown in the simulation studies is only for visualization purpose. Because the MEG sensors are much more sensitive to tangential sources than to radial ones, the orientation of each of the three dipoles in our simulation was arbitrarily set but lay on the plane tangential to the head conductor sphere. Strengths of the red, blue, and green dipoles were all zeros from $-1 \mathrm{~s}$ to $0 \mathrm{~s}$ and were 10,50 , and $50 \mathrm{nAm}$,
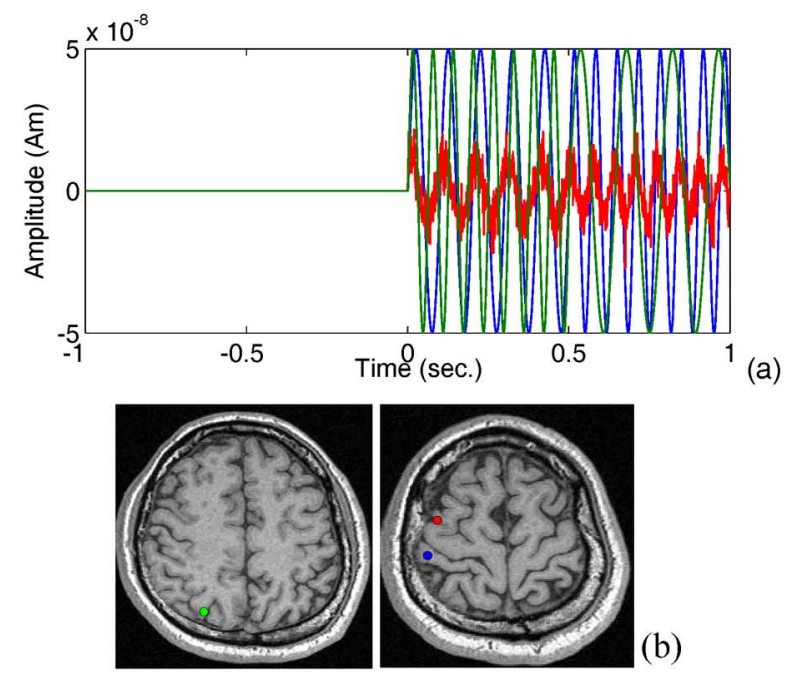

(b)
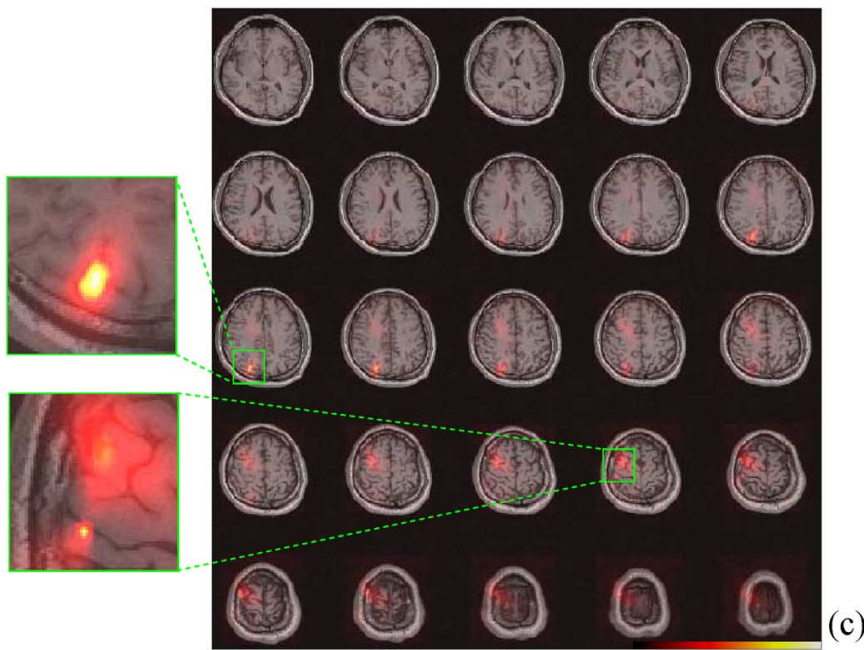

Fig. 1. Ground truth and estimated sources of three dipoles in a simulation study. This figure illustrates (a) the temporal profiles of these three dipole as well as (b) their positions superimposed on a head MRI. The F-statistic map calculated by using the proposed MCB method is tomographically shown in (c) with two regions around the estimated sources enlarged.

respectively, from $0 \mathrm{~s}$ to $1 \mathrm{~s}$. Frequencies of the temporal profiles for the red and blue dipoles were the same, but were different from that for the green dipole. Zero-mean Gaussian random noise with standard deviation $5 \mathrm{nAm}$ was added to the temporal profile of the red dipole. Correlation coefficient of the temporal profiles of the red and blue dipoles was about 0.58 . Temporal profile of the green dipole was not correlated to those of the other two dipoles (with correlation coefficient values about 0.03 to the red dipole and 0 to the blue one). In addition to these three dipoles, 3000 random dipoles were uniformly distributed throughout the brain region to simulate the nontask-related activities. The strength of each random dipole was drawn from a zero-mean Gaussian random number with standard deviation $10 \mathrm{nAm}$. Based on the forward MEG solutions, the simulated magnetic signals were then calculated at a 1 -ms interval from $-1 \mathrm{~s}$ to $1 \mathrm{~s}$. Sensor noises with variance estimated from the empty room recordings of the MEG system were also added to the simulated signals. The simulated signals 


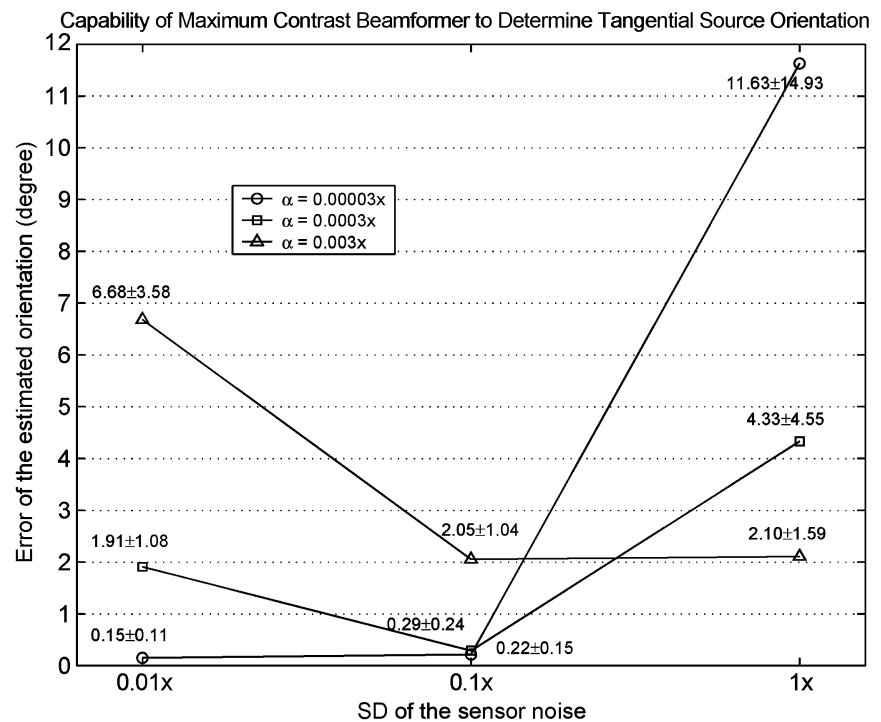

Fig. 2. Illustration of the mean and the standard deviation (SD) of the orientation estimation error (mean $\pm \mathrm{SD}$ ) for different regularization value $\alpha$ and varying sensor noise levels.

were then processed by a bandpass filter $(1 \sim 20 \mathrm{~Hz})$ followed by a baseline correction procedure.

1) Accuracy of Source Localization: The proposed MCB method was used to calculated the F-statistic map for the simulated magnetic signals, in which the time windows of the active and control states were $0 \sim 1 \mathrm{~s}$ and $-1 \sim 0 \mathrm{~s}$, respectively. Fig. 1(c) illustrates the obtained F-statistic map overlaid on MRI slices. The scanning proceeded voxel-by-voxel and the red, blue, and green dipoles were located in the scanning space when they were used to simulate the MEG signals. Obviously we can find three sources in the obtained F-statistic map and the three peak F-value locations accurately match with the ground-truth locations of these three dipoles.

Among the three dipoles, the green dipole was the most focal and significant one revealed in the F-statistic map because its dipole strength was larger and its temporal waveforms were not correlated to others. Although the blue dipole had the same dipole strength as the green one, but it was close to another correlated source, the red dipole, such that the $\mathrm{F}$ statistic for the blue dipole was smaller than that for the green one, as discussed in [15]. This phenomenon was even worse for the red dipole in which the F statistic was diversely distributed, as shown in Fig. 1(c), due to its relative small dipole strength.

2) Accuracy of Dipole Orientation Estimation: The abovementioned procedure was repeated to simulate the magnetic signals for the assessment of dipole orientation estimation accuracy by using the proposed MCB method. Only the blue dipole, instead of the three dipoles concurrently, was engaged in this case. Ninety dipole orientations were regularly sampled on the tangential plane for the blue dipole to generate ninety sets of magnetic signals. Fig. 2 illustrates the accuracy performance of orientation estimation by using the proposed method. The circle, square, and triangle marks indicate the results when the regularization parameters were set to be $0.00003,0.0003$, and 0.003 times the maximum eigenvalue of the active state covariance matrix, respectively. The horizontal axis represents the levels of sensor noise in the simulated data, ranging from 0.01 to 1 times the standard deviation of the empty room measurements. The vertical axis represents the average of orientation estimation errors for ninety trials. From this figure, we can see that the average of the orientation estimation errors can be under $2.1^{\circ}$ when the regularization is set appropriately. When the recorded signals are of high SNR, that is, the sensor noise is low, a smaller regularization value can achieve better accuracy. Nevertheless, it remains a challenging issue to determine a proper regularization value in the beamforming method.

\section{B. Phantom}

An MEG phantom (Neuromag Ltd., Finland) was used to evaluate the localization accuracy of the MCB. Four-head position indicator (HPI) coils fixed on the phantom were engaged to obtain the position of the phantom with respect to the sensor device. Sixteen fixed current dipoles located on two orthogonal planes were activated sequentially to generate the magnetic fields. The current strength of each dipole was set to be $50 \mathrm{nAm}$. For each dipole, 50 trials were recorded by the MEG system at a sampling rate of $1000 \mathrm{~Hz}$ and then averaged according to the activation onset time. The averaged data were then processed by a bandpass filter $(7.5 \mathrm{~Hz}$ to $35 \mathrm{~Hz})$ followed by a baseline correction procedure. We chose the time window from $30 \mathrm{~ms}$ to $90 \mathrm{~ms}$ as the active state to calculate the covariance matrix $\mathbf{C}_{a}$ (and C). Empty room measurements were used to calculated as the control state covariance matrix $\mathbf{C}_{c}$. The regularization value was set to be 0.0003 times the maximum eigenvalue of the covariance matrix $\mathbf{C}_{a}$.

The MCB method was applied to calculate the F-statistic map and the position with peak value was located as the estimated dipole position. The Euclidean distance between the ground truth and the estimated position was calculated as the position estimation error. The average of position estimation error for the engaged sixteen dipoles was $1.6381 \mathrm{~mm}$ with standard deviation $0.4971 \mathrm{~mm}$, which is similar to those in the literature [30], [31]. The average of orientation estimation error was $1.9362^{\circ}$ with standard deviation $0.7054^{\circ}$. These results clearly demonstrate the effectiveness and accuracy of the proposed MCB method.

\section{Self-Paced Finger Movement}

In this study, the movement-evoked magnetic fields of one right-handed healthy subject were acquired. The subject was asked to sit in a comfortable chair with eyes open in a magnetically shielded room. The subject performed self-paced, brisk left/right index finger extension (finger lifting) movements at irregular time intervals longer than $8 \mathrm{~s}$. Finger extension was immediately followed by brief muscle relaxation. The commencement of finger movement was registered using an optical pad and the trigger time was defined as onset time $0 \mathrm{~ms}$.

Vertical and horizontal electrooculogram (EOG) were recorded to obtain the EOG-free epochs which were not contaminated by eye movements and/or blinks. About 100 EOG-free epochs of MEG measurements with a sampling rate of $250 \mathrm{~Hz}$ were acquired and then averaged according to the trigger onsets followed by bandpass filtering $(3 \sim 35 \mathrm{~Hz})$ and baseline correction. Four HPI coils, two attached on the forehead and 


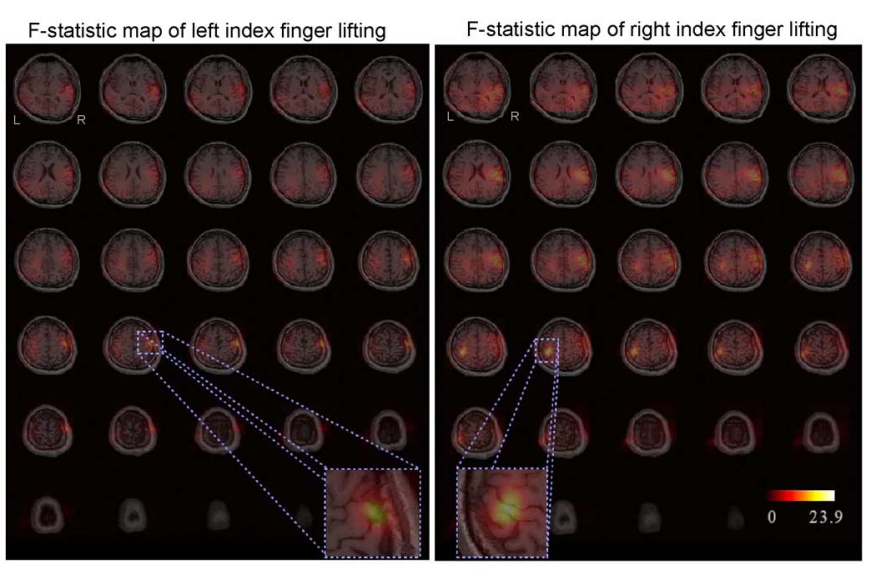

Fig. 3. F-statistic map of the estimated sources of left and right index finger movement-evoked fields using the MCB method.

two behind the ears, were used to locate the head of the subject as regard to the sensor array before data recordings.

The MRI volume of size $256 \times 256 \times 128$ was scanned with a Siemens MR system where the MR-RAGE pulse sequence was performed with $\mathrm{TR}=1800 \mathrm{~ms}$, TE $=4.38 \mathrm{~ms}$, $\mathrm{TI}=$ $1100 \mathrm{~ms}$, and $\mathrm{FOV}=230 \times 230 \times 192 \mathrm{~mm}^{3}$. The coordinate systems between the MR volume and MEG device were co-registered by means of locating three landmarks (left pre-auricular, right pre-auricular, and nasion points) in both systems.

The proposed method was again employed to map the sources of movement-evoked fields. The active state was defined as the duration from $-120 \mathrm{~ms}$ before onset to $360 \mathrm{~ms}$ after onset. The covariance matrix of the control state was estimated from the empty room recordings of $40 \mathrm{~s}$ in order to obtain a large amount of sample data for good estimate of the covariance matrix. Fig. 3 illustrates the calculated F-statistic map. In this figure, the position of the maximum F-value locates around the hand area of the primary sensorimotor cortex in the contralateral hemisphere for both left/right finger movement tasks.

\section{DISCUSSION}

\section{A. Importance of Accurate Orientation Estimation}

Dipole orientation estimation is a critical issue in scalar-type beamforming methods [22]-[24]. The spatial filter calculated for a dipole with inaccurate orientation fails to correctly estimate the neuronal activity, particularly when the specificity of the spatial filter is high, that is, when the regularization parameter $\alpha$ is small. In this case, the beamformer may not reveal the true significance level of task-related activities at this probed position. Below we discuss this issue theoretically and experimentally.

The following theorem describes that under certain assumptions, the source will be missed by the optimal solution of the scalar beamformer even when the true source location is targeted.

Theorem 1: Assume that there is no noise and the MEG signals are originated from a single source with dipole parameters $\theta=\left\{\mathbf{r}_{\mathbf{q}}, \mathbf{q}\right\}$ in the brain, where the source activity $s_{\theta}(t)$ is with zero mean and non-zero power. Consider the calculation of a scalar spatial filter $\mathbf{w}_{\theta^{\prime}}$ targeted at the true source location, where $\theta^{\prime}=\left\{\mathbf{r}_{\mathbf{q}}, \mathbf{q}^{\prime}\right\}$ represents the dipole with location $\mathbf{r}_{\mathbf{q}}$ and orientation $\mathbf{q}^{\prime}$ deviating from the true source orientation $\mathbf{q}$, $\mathbf{q}^{\prime} \neq \mathbf{q}$. Then there exists an optimal solution of the scalar spatial filter $\hat{\mathbf{w}}_{\theta^{\prime}}$ with ultimate spatial specificity $(\alpha=0)$, based on the unit-gain constraint and minimum variance criterion, such that the filter output of $\hat{\mathbf{w}}_{\theta^{\prime}}$ is zero.

Proof: Since there is no noise, the MEG signals can be measured as

$$
\mathbf{m}(t)=s_{\theta}(t) \mathbf{l}_{\theta}=s_{\theta}(t) \mathbf{L}_{\mathbf{r}_{\mathbf{q}}} \mathbf{q} .
$$

Because the mean of the source activity $s_{\theta}(t)$ and $\alpha$ are both zeros, (5) can be rewritten as

$$
\begin{aligned}
\hat{\mathbf{w}}_{\theta^{\prime}} & =\arg \min _{\mathbf{w}_{\theta^{\prime}}} E\left\{\left\|\mathbf{w}_{\theta^{\prime}}^{T} \mathbf{m}(t)\right\|^{2}\right\} \quad \text { subject to } \mathbf{w}_{\theta^{\prime}}^{T} \mathbf{l}_{\theta^{\prime}}=1 \\
& =\arg \min _{\mathbf{w}_{\theta^{\prime}}} E\left\{\left\|s_{\theta}(t) \mathbf{w}_{\theta^{\prime}}^{T} \mathbf{l}_{\theta}\right\|^{2}\right\} \quad \text { subject to } \mathbf{w}_{\theta^{\prime}}^{T} \mathbf{l}_{\theta^{\prime}}=1 \\
& =\arg \min _{\mathbf{w}_{\theta^{\prime}}}\left\{\sigma_{\theta}^{2} \mathbf{w}_{\theta^{\prime}}^{T} \mathbf{l}_{\theta} \mathbf{l}_{\theta}^{T} \mathbf{w}_{\theta^{\prime}}\right\} \quad \text { subject to } \mathbf{w}_{\theta^{\prime}}^{T} \mathbf{l}_{\theta^{\prime}}=1,
\end{aligned}
$$

where $\sigma_{\theta}^{2}$ is the non-zero power of the source activity. Since the matrix $\mathbf{l}_{\theta} \mathbf{l}_{\theta}^{T}$ is real symmetric, $\mathbf{w}_{\theta^{\prime}}^{T} \mathbf{l}_{\theta} \mathbf{l}_{\theta}^{T} \mathbf{w}_{\theta^{\prime}} \geq 0$ is true for all $\mathbf{w}_{\theta^{\prime}} \in \Re^{N}$. Obviously the vector $\mathbf{w}_{\theta^{\prime}}$ satisfying the condition of $\mathbf{w}_{\theta^{\prime}}^{T} \mathbf{l}_{\theta}=0$ achieves the minimum value of the objective function $E\left\{\left\|\mathbf{w}_{\theta^{\prime}}^{T} \mathbf{m}(t)\right\|^{2}\right\}$. Combined with the unit-gain constraint $\mathbf{w}_{\theta^{\prime}}^{T} \mathbf{l}_{\theta^{\prime}}=1$, the vector which satisfies both the conditions of $\mathbf{w}_{\theta^{\prime}}^{T} \mathbf{l}_{\theta}=0$ and $\mathbf{w}_{\theta^{\prime}}^{T} \mathbf{l}_{\theta^{\prime}}=1$ is the optimal solution of the spatial filter, $\hat{\mathbf{w}}_{\theta^{\prime}}$. As a result, the $\mathrm{F}$ statistic can be calculated as

$$
\begin{aligned}
F_{\theta^{\prime}} & =\frac{E\left\{\left\|\hat{\mathbf{w}}_{\theta^{\prime}}^{T} \mathbf{m}(t)\right\|^{2}\right\}}{E\left\{\left\|\hat{\mathbf{w}}_{\theta^{\prime}}^{T} \mathbf{m}_{c}(t)-E\left\{\hat{\mathbf{w}}_{\theta^{\prime}}^{T} \mathbf{m}_{c}(t)\right\}\right\|^{2}\right\}} \\
& =\frac{E\left\{\left\|s_{\theta}(t) \hat{\mathbf{w}}_{\theta^{\prime}}^{T} \mathbf{l}_{\theta}\right\|^{2}\right\}}{E\left\{\left\|\hat{\mathbf{w}}_{\theta^{\prime}}^{T} \mathbf{m}_{c}(t)-E\left\{\hat{\mathbf{w}}_{\theta^{\prime}}^{T} \mathbf{m}_{c}(t)\right\}\right\|^{2}\right\}} \\
& =0 .
\end{aligned}
$$

That is, the filter output $\hat{\mathbf{w}}_{\theta^{\prime}}^{T} \mathbf{m}(t)$ and $\mathrm{F}$ statistic $F_{\theta^{\prime}}$ are both zeros.

From the geometric point of view, $\mathbf{w}_{\theta^{\prime}}^{T} \mathbf{l}_{\theta}=0$ represents the hyperplane $\pi_{1}$ that has the normal vector $\mathbf{l}_{\theta}$ and passes through the origin $\mathbf{O}$, as shown in Fig. 4. Similarly, $\mathbf{w}_{\theta^{\prime}}^{T} \mathbf{l}_{\theta^{\prime}}=1$ represents the hyperplane $\pi_{2}$ with the normal vector $\mathbf{l}_{\theta^{\prime}}$ and with distance $1 /\left\|\mathbf{l}_{\theta^{\prime}}\right\|$ to the origin. Therefore, the optimal solution of $\hat{\mathbf{w}}_{\theta^{\prime}}$ is the intersection line of these two hyperplanes $\pi_{1}$ and $\pi_{2}$. This line can be represented by

$$
\hat{\mathbf{w}}_{\theta^{\prime}}=c_{1} \mathbf{n}_{1}+c_{2} \mathbf{n}_{2}+\lambda \mathbf{n}_{1} \times \mathbf{n}_{2}
$$

where $\mathbf{n}_{1}=\mathbf{l}_{\theta} /\left\|\mathbf{l}_{\theta}\right\|, \mathbf{n}_{2}=\mathbf{l}_{\theta^{\prime}} /\left\|\mathbf{l}_{\theta^{\prime}}\right\|, c_{1}=-\cos \gamma /$ $\left(\left\|\mathbf{1}_{\theta^{\prime}}\right\| \sin ^{2} \gamma\right), c_{2}=1 /\left(\left\|\mathbf{1}_{\theta^{\prime}}\right\| \sin ^{2} \gamma\right), \gamma$ is the angle between $l_{\theta}$ and $l_{\theta^{\prime}}$, and $\lambda$ is the parameter of the line.Notice that this line exists only when the angle $\gamma \neq 0$, or equivalently, $\mathbf{q}^{\prime} \neq \mathbf{q}$. Fig. 4 illustrates the geometric relationship between these two planes and the optimal solution of the spatial filter. 


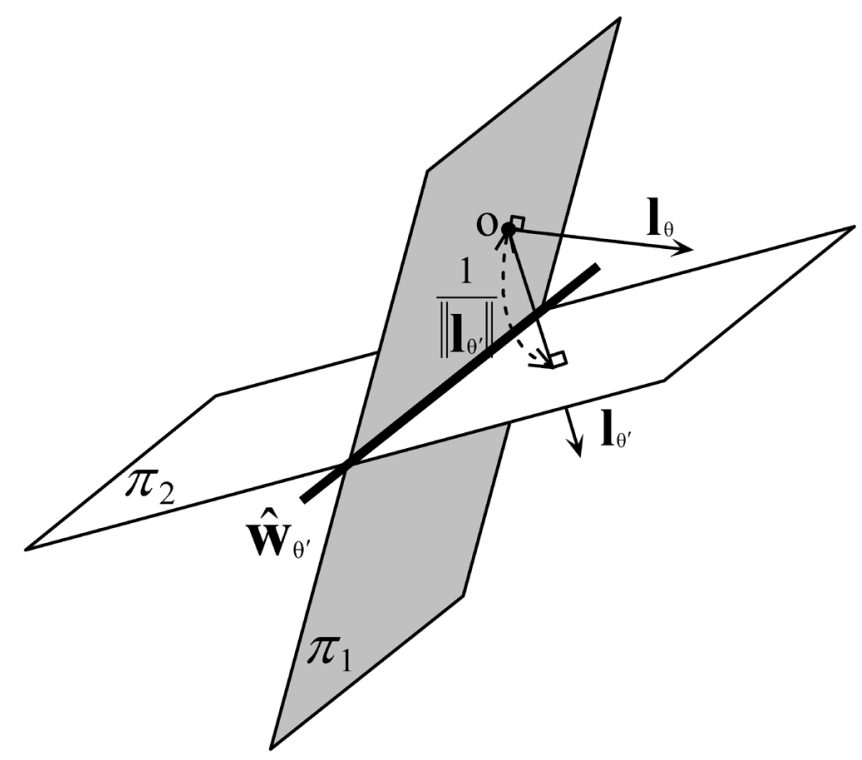

Fig. 4. Optimal solution of the spatial filter in a special case. Under the assumptions that there is no noise and the MEG signals are originated from a single dipole source with lead field $\mathbf{l}_{\theta}$, the optimal solution of the ultimate-specificity spatial filter $\hat{\mathbf{w}}_{\theta^{\prime}}$ for dipole $\theta^{\prime}$ is the intersection of two hyperplanes, $\mathbf{w}_{\theta^{\prime}}^{T} \mathbf{l}_{\theta}=0\left(\pi_{1}\right)$ and $\mathbf{w}_{\theta^{\prime}}^{T} \mathbf{l}_{\theta^{\prime}}=1\left(\pi_{2}\right)$, where $\mathbf{l}_{\theta^{\prime}}$ is the lead field of dipole $\theta^{\prime}$.

According to the above theorem, the source will be missed by an ultimate-specificity scalar beamformer with deviated dipole orientation when noise-free MEG measurements originate from a single dipole source. Practically, there exist various kinds of noise in the MEG measurements, including physiological artifacts, instrumentation perturbation, and environment interference. To accommodate the spatial filter to the influence of the noise, the regularization parameter $\alpha$ can be used to control the norm of the spatial filter. Increasing the value of $\alpha$ will prefer the spatial filter with lower norms; thus, can increase the noise resistance capability of the spatial filter at the expense of degrading its spatial specificity. Consequently, the spatial filter does not lie on the intersection line such that the targeted dipole source will not be totally missed in this case. However, leakage from the sensor noise and all the sources other than the targeted one inevitably contribute to the spatial filter output. Accurate dipole orientation is very essential to differentiate the response of the targeted source from those of noise and other sources.

We generated a set of simulation data to investigate the issue that how the dipole orientation influence the performance of scalar beamformers. The procedure of generating simulation data described in Section III-A was repeated to produce the magnetic signals. The red and blue dipoles were both engaged and the green dipole was discarded in this case, as shown in Fig. 5(a). The orientation of the red dipole was aligned to the $y$ axis. The blue dipole was oriented to have included angles of $54.7^{\circ}$ from each of the three coordinate axes. The top and bottom parts of Fig. 5(b) show the F-statistic map calculated by using the proposed MCB method and the pseudo-Z statistic map calculated by using the scalar beamformer with dipole orientation specified as the $y$ axis, respectively. The regularization parameter $\alpha$ was set to be 0.0003 for both methods.

Obviously, the proposed MCB method can accurately estimate the dipole orientation and produce two focal distributions

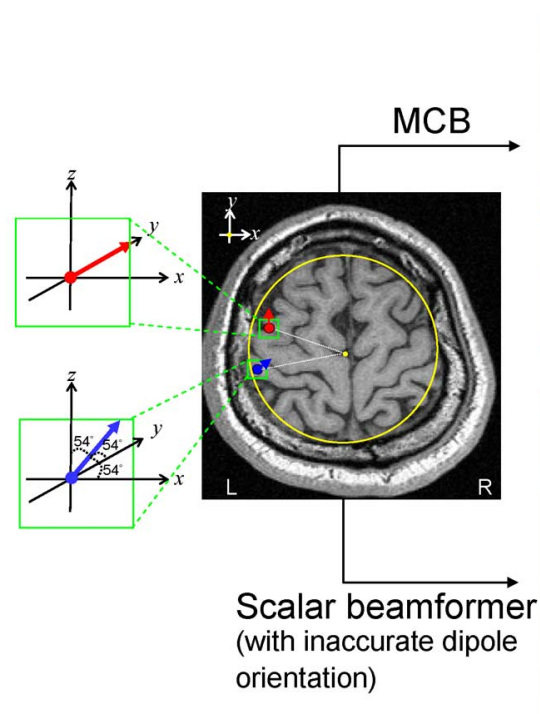

(a)

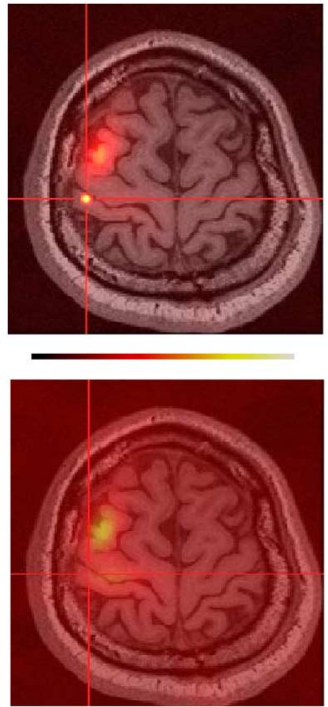

(b)
Fig. 5. Effect of dipole orientation on scalar beamformer. (a) Two dipoles for generating the simulated MEG signals. (b) F-statistic map calculated by using the proposed MCB method (top) and the pseudo- $Z$ statistic map calculated by the scalar beamformer with orientation specified as the $y$ axis (bottom).

that match these two dipole sources. Besides, the blue dipole has a more focused distribution than the red one because the former has higher SNR. The scalar beamformer also obtained strong pseudo-Z statistic around the red dipole position because the orientation is correctly specified as the true source orientation, the $y$ axis. However, the activity distribution around the blue dipole position is not significant because the specified orientation is largely deviated to the blue dipole.

\section{B. Comparison With LCMV Beamformer}

As noted in [23], the scalar beamformer achieves higher output SNR than that of the vector beamformer when the probed dipole orientation is accurately matched to the source dipole orientation. However, the performance of the scalar beamformer degrades when the probed dipole orientation deviates from the source. Because the vector beamformer is independent of the dipole orientation, we need to evaluate the performance of the scalar beamformer, relative to that of the vector beamformer, with respect to the accuracy of dipole orientation estimation. The experiment for this purpose is described below.

The procedure of generating simulation data described in Section III-A was repeated to produce the magnetic signals. In this case, the blue dipole was engaged while the red and green dipoles were discarded. Targeted at the ground-truth location, the $\mathrm{F}$ values were calculated by the scalar beamformer and LCMV beamformer where $\alpha$ was set to be 0.003 times the maximum eigenvalue of the covariance matrix $\mathbf{C}$. For the scalar beamformer, the $\mathrm{F}$ value decreases when the deviation of the probed dipole orientation becomes large, as indicated by the square marks in Fig. 6(a). When the dipole orientation deviation is below around $6^{\circ}$, the scalar beamformer produces higher $\mathrm{F}$ values than the LCMV method does, as indicated by the dotted line in Fig. 6(a). However, the performance of 

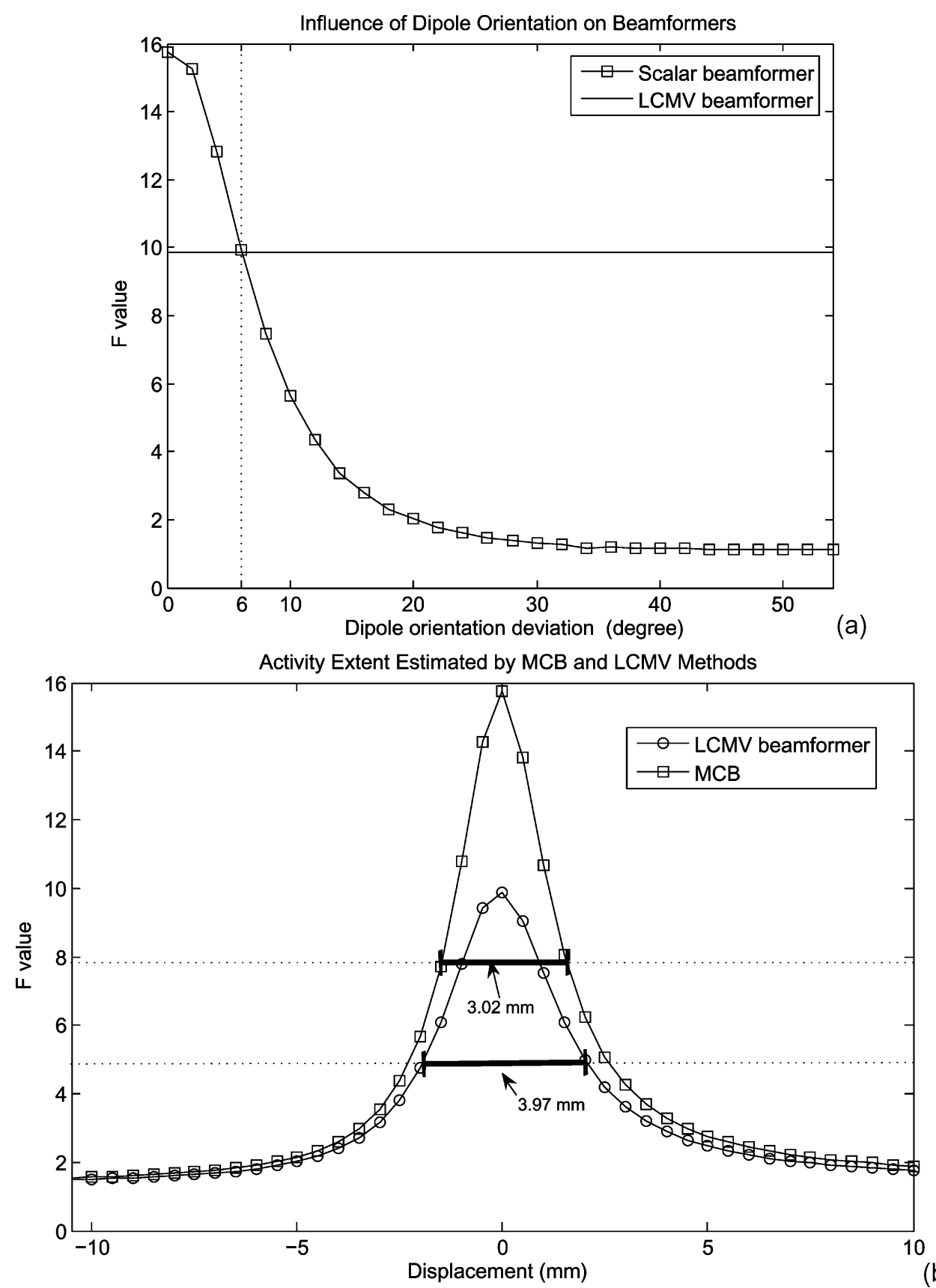

Fig. 6. Performance comparison between MCB and LCMV methods. (a) F value obtained by the scalar beamformer degrades when dipole orientation deviation becomes large, as indicated by the square marks. It drops below what LCMV beamformer obtains when the orientation deviation is larger than around $6^{\circ}$ (the dotted line). In our experiments, the proposed MCB method can achieve around $2^{\circ}$ of orientation estimation error and, thus, can obtain superior $\mathrm{F}$ values. (b) Crosssectional spatial extents of the source activity estimated by the MCB (square marks) and LCMV (circle marks) methods and the widths at half-peak values are $3.02 \mathrm{~mm}$ and $3.97 \mathrm{~mm}$, respectively.

the scalar beamformer drops below that of the LCMV method when the dipole orientation deviation is larger than $6^{\circ}$. In our simulation and phantom studies described in Section III, the proposed MCB method can achieve orientation estimation accuracy around $2^{\circ}$, which is much less than the turning point of $6^{\circ}$.

Fig. 6(b) illustrates the cross-sectional spatial extents of the source activities estimated by the MCB and LCMV methods. The horizontal axis represents the displacement between the true source location and the probed dipole location along the depth. Targeting at the true source location, the peak $\mathrm{F}$ values estimated by the MCB and LCMV methods are 15.75 and 9.86, respectively. The width at half-peak $\mathrm{F}$ value obtained by the MCB method is $3.02 \mathrm{~mm}$ and that obtained by the LCMV method is $3.97 \mathrm{~mm}$. The width ratio of LCMV to MCB is 1.31, which is close to that of LCMV to SAM (which is $\sqrt{2}$ ), as reported in [23]. These results demonstrate the the superiority of the MCB method in imaging brain activities because it can achieve high $\mathrm{F}$ value and focal spatial extent of the estimated brain activity distribution. Notice that the experiments presented here were performed on a 204-channel sensor array. Performance of the MCB method will degrade 


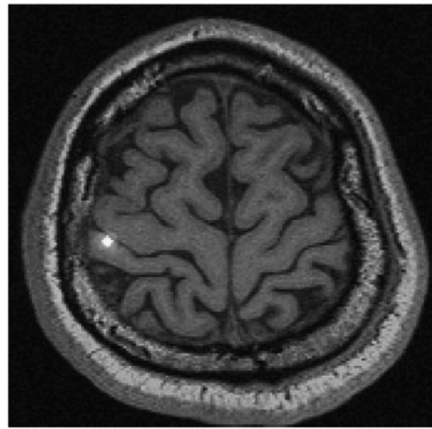

(a)

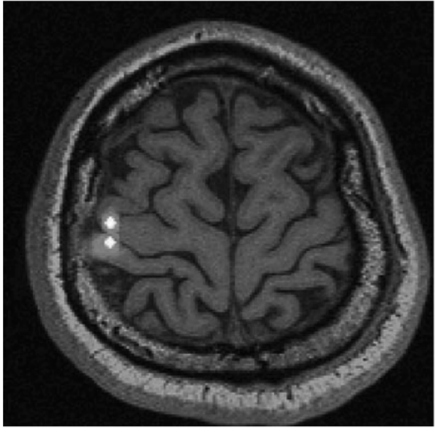

(b)
Fig. 7. Statistic maps of source activities estimated with measurements of different control states. (a) F-statistic map of source activity when the dipole activity contributes to the control-state measurements. Only the blue dipole is revealed due to its high activity ratio between the active and control states. (b) F-statistic map when the control-state measurements only contain sensor noise. Two dipoles are revealed because they both have large activities during the active state compared to the sensor noise.

relative to the LCMV method when the sensor number increases [23].

\section{Influence of Measurements in Control State}

The MCB method calculates the F value, which is the variance ratio of the filtered activity obtained from the active state to that obtained from the control state. Choices of control state affect the calculated $\mathrm{F}$ value as well as the resulted distribution of neuronal activation. We performed the following experiment by using simulation data to investigate this issue. The procedure of generating simulation data described in Section III-A was repeated to produce the magnetic signals. The blue dipole was engaged with its position depicted in Fig. 1(b). In this case, the blue source dipole was activated only in the active state (that is, from 0 to $1 \mathrm{~s}$ ) with strength $30 \mathrm{nAm}$. Another engaged source dipole was located on the same horizontal plane as and at a distance of $7.2 \mathrm{~mm}$ of the blue dipole. This additional dipole with strength $50 \mathrm{nAm}$ was activated from -1 to $1 \mathrm{~s}$, that is, throughout both the control and active states.

The simulated MEG recordings in the active and control states were used to calculate the corresponding covariance matrices, $\mathbf{C}_{a}$ and $\mathbf{C}_{c}$, respectively. Then, the F-statistic map of source activity was calculated by the MCB method where $\alpha$ was set to be 0.003 times the maximum eigenvalue of the covariance matrix C. As shown in Fig. 7(a), the blue dipole is clearly revealed because there is significant contrast of source activity between the active and control states. On the other hand, the other dipole is not revealed due to its relatively low activity contrast between active and control states, compared to the blue one. Therefore, this choice of control state is useful for dual-state experiments.

If the measurements in the control state only contain the sensor noises that are independent and identically distributed, the covariance matrix $\mathbf{C}_{c}$ is the identity matrix $\mathbf{I}$ times the variance of the sensor noise. In this case the $F$ value becomes the output SNR (pseudo-Z statistic) in the method proposed by Sekihara et al. [22]. As shown in Fig. 7(b), two dipoles are revealed because their activities during the active state are both large compared to the sensor noise.

\section{Computation Time}

In addition to its capability of estimating dipole orientation accurately, the MCB method is also superior in computation efficiency due to its closed-form solution of dipole orientation. Only the matrix inverse and eigenproblem for a $3 \times 3$ matrix are involved in the orientation estimation procedure, which is apparently very efficient. In the SAM method, the dipole orientation is determined by searching in the solution space according to the calculated value of pseudo- $Z$ statistic. The involved optimization procedure is inherently time-consuming and may trap in the local minimum.

For efficiency comparison, we applied both the MCB and SAM methods to determine the dipole orientation for the simulation data set used in Section III-A. We tackled the search problem of the SAM method by exhaustively probing the solution space of dipole orientation at an interval of $5^{\circ}$. The solution space can be either restricted on the two-dimensional (2-D) tangential plane or the three-dimensional (3-D) space in general. The comparison did not include the vector beamformer methods because there is no orientation determination procedure involved in these methods.

The comparison was performed on a computer equipped with an AMD Athlon XP $3000+$ CPU (2.17 GHz) and $512 \mathrm{MB}$ RAM. The dipole orientation determination procedure was repeated 100000 times and the total computation time was measured for each method. The SAM method required $4.0 \mathrm{~s}$ and $282.3 \mathrm{~s}$ to probe 36 and 2592 candidates on the 2-D plane and in the 3-D space, respectively. The proposed MCB method used only $1.3 \mathrm{~s}$, which is more than three times and 200 times faster than the SAM method in 2-D and 3-D cases, respectively.

\section{CONCLUSION}

In this paper, we have proposed a novel beamforming approach, the MCB method, for statistically mapping the brain activity from MEG recordings. Based on the maximum contrast criterion, the proposed method calculate a spatial filter that can maximize the significance level, F statistic, indicating the variance ratio of filtered activities between two specified time windows. The spatial filter is calculated according to the dipole orientation, which can be optimally determined very efficiently in a closed-form manner. According to our experiments, we have clearly demonstrated the effectiveness, efficiency, and accuracy of the proposed method.

\section{ACKNOWLEDGMENT}

The authors would like to thank anonymous reviewers for their insightful and constructive comments to help improve the quality of this paper.

\section{REFERENCES}

[1] J. Sarvas, "Basic mathematical and electromagnetic concepts of the biomagnetic inverse problem," Phys. Med. Biol., vol. 32, no. 1, pp. 11-22, Jan. 1987.

[2] J. C. Mosher, R. M. Leahy, and P. S. Lewis, "EEG and MEG: forward solutions for inverse methods," IEEE Trans. Biomed. Eng., vol. 46, no. 3, pp. 245-259, Mar. 1999

[3] M. Hämäläinen, R. Hari, R. J. Ilmoniemi, J. Knuutila, and O. V. Lounasmaa, "Magnetoencephalography-theory, instrumentation, and applications to noninvasive studies of the working human brain," Rev. Modern Phys., vol. 65, no. 2, pp. 413-497, Apr. 1993.

[4] S. Baillet, J. C. Mosher, and R. M. Leahy, "Electromagnetic brain mapping," IEEE Signal Process. Mag., vol. 18, no. 6, pp. 14-30, Nov. 2001. 
[5] M. Huang, C. J. Aine, S. Supek, E. Best, D. Ranken, and E. R. Flynn, "Multi-start downhill simplex method for spatio-temporal source localization in magnetoencephalography," Electroencephalogr. Clin. Neurophysiol. (Evoked Potentials Section), vol. 108, no. 1, pp. 32-44, Jan. 1998.

[6] J. C. Mosher, P. S. Lewis, and R. M. Leahy, "Multiple dipole modeling and localization from spatio-temporal MEG data," IEEE Trans. Biomed. Eng., vol. 39, no. 6, pp. 541-557, Jun. 1992.

[7] J. C. Mosher and R. M. Leahy, "Recursive MUSIC: a framework for EEG and MEG source localization," IEEE Trans. Biomed. Eng., vol. 45, no. 11 , pp. 1342-1354, Nov. 1998.

[8] — , "Source localization using recursively applied and projected (RAP) MUSIC," IEEE Trans. Signal Process., vol. 47, no. 2, pp. 332-340, Feb. 1999.

[9] M. Hämäläinen and R. Ilmoniemi, "Interpreting magnetic fields of the brain: minimum norm estimates," Med. Biol. Eng. Comput., vol. 32, no. 1, pp. 35-42, Jan. 1994.

[10] K. Matsuura and Y. Okabe, "Selective mimimum-norm solution of the biomagnetic inverse problem," IEEE Trans. Biomed. Eng., vol. 42, no. 6, pp. 608-615, Jun. 1995.

[11] K. Uutela, M. Hämäläinen, and E. Somersalo, "Visualization of magnetoencephalographic data using minimum current estimates," NeuroImage, vol. 10, no. 2, pp. 173-180, Aug. 1999.

[12] G. R. Barnes and A. Hillebrand, "Statistical flattening of MEG beamformer images," Hum. Brain Mapp., vol. 18, no. 1, pp. 1-12, Jan. 2003.

[13] H. Krim and M. Viberg, "Two decades of array signal processing research: the parametric approach," IEEE Signal Process. Mag., vol. 13, no. 4, pp. 67-94, Jul. 1996

[14] H. Cox, R. M. Zeskind, and M. M. Owen, "Robust adaptive beamforming," IEEE Trans. Acoust., Speech, Signal Process., vol. ASSP-35, no. 10, pp. 1365-1376, Oct. 1987.

[15] B. D. Van Veen, W. van Drongelen, M. Yuchtman, and A. Suzuki, "Localization of brain electrical activity via linearly constrained minimum variance spatial filter," IEEE Trans. Biomed. Eng., vol. 44, no. 9, pp. 867-879, Sep. 1997.

[16] S. E. Robinson and J. Vrba, "Functional neuroimaging by synthetic aperture magnetometry (SAM)," in Recent Advances in Biomagnetism. Sendai, Japan: Tohoku Univ. Press, 1999, pp. 302-305.

[17] K. Sekihara, S. S. Nagarajan, D. Poeppel, A. Marantz, and Y. Miyashita, "Reconstructing spatio-temporal activities of neural sources using an MEG vector beamformer technique," IEEE Trans. Biomed. Eng., vol. 48, no. 7, pp. 760-771, Jul. 2001.

[18] J. Gross, J. Kujala, M. Hämäläinen, L. Timmermann, A. Schnitzler, and R. Salmelin, "Dynamic imaging of coherent sources: studying neural interactions in the human brain," Proc. Nat. Acad. Sci., vol. 98, no. 2, pp. 694-699, Jan. 2001.

[19] J. Gross, L. Timmermann, J. Kujala, R. Salmelin, and A. Schnitzlera, "Properties of MEG tomographic maps obtained with spatial filtering," NeuroImage, vol. 19, no. 4, pp. 1329-1336, Aug. 2003.

[20] W. C. Gaetz and D. O. Cheyne, "Localization of human somatosensory cortex using spatially filtered magnetoencephalography," Neuroscience Letter, vol. 340, no. 3, pp. 161-164, Apr. 2003.

[21] K. D. Singh, G. R. Barnes, and A. Hillebrand, "Group imaging of task-related changes in cortical synchronisation using nonparametric permutation testing," NeuroImage, vol. 19, no. 4, pp. 1589-1601, Aug. 2003.

[22] K. Sekihara, S. S. Nagarajan, D. Poeppel, and A. Marantz, "Asymptotic SNR of scalar and vector minimum-variance beamformers for neuromagnetic source reconstruction," IEEE Trans. Biomed. Eng., vol. 51, no. 10 , pp. 1726-1734, Oct. 2004.

[23] J. Vrba and S. E. Robinson, "Differences between synthetic aperture magnetometry (SAM) and linear beamformers," in Proc. 12th Int. Conf. Biomagnetism, Espoo, Finland, 2000, pp. 681-684.

[24] A. Hillebrand and G. R. Barnes, "The use of anatomical constraints with MEG beamformers," NeuroImage, vol. 20, no. 4, pp. 2302-2313, Dec. 2003.

[25] H.-Y. Liu, Y.-S. Chen, L.-F. Chen, and J.-C. Hsieh, "Statistical mapping of cortical activities using minimum-variance maximum-discrimination spatial filtering," Int. J. Bioelectromagn., vol. 7, no. 2, pp. 104-107, 2005.

[26] A. N. Tikhonov and V. A. Arsenin, Solutions of Ill-Posed Problems. Pittsford, NY: Winston, 1977.

[27] J. Gross and A. A. Ioannides, "Linear transformations of data space in MEG," Phys. Med. Biol., vol. 44, no. 8, pp. 2081-2097, Aug. 1999.

[28] D. C. Lay, Linear Algebra and its Applications, 2nd ed. Reading, MA: Addison Wesley, 1998.

[29] J. V. Uspensky, Theory of Equations. New York: McGraw-Hill, 1958.
[30] E. Menninghaus, B. Lutkenhoner, and S. Gonzalez, "Localization of a dipolar source in a skull phantom: realistic versus spherical model," IEEE Trans. Biomed. Eng., vol. 41, no. 10, pp. 986-989, Oct. 1994.

[31] R. M. Leahy, J. C. Mosher, M. E. Spencer, M. X. Huang, and J. D. Lewine, "A study of dipole localization accuracy for MEG and EEG using a human skull phantom," Electroencephalogr. Clin. Neurophysiol., vol. 107, no. 2, pp. 159-173, Aug. 1998.

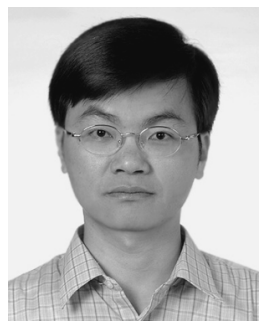

Yong-Sheng Chen (S'01-M'03) received the B.S. degree in computer and information science from National Chiao Tung University, Hsinchu, Taiwan, in 1993. He received the M.S. a Ph.D. degrees in computer science and information engineering from National Taiwan University, Taiwan, in 1995 and 2001, respectively.

$\mathrm{He}$ is currently an Assistant Professor in the Department of Computer Science, National Chiao Tung University. His research interests include biomedical signal processing, medical image processing, and computer vision.

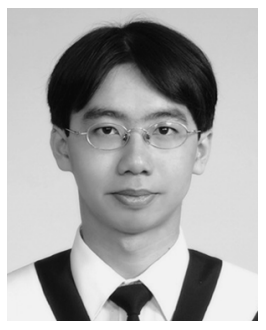

Chih-Yu Cheng received the B.S. degree in engineering science from National Cheng Kung University, Tainan, Taiwan, in 2003 and the M.S. degree in computer science and information engineering from National Chiao Tung University, Hsinchu, Taiwan, in 2005. The research topic of his master thesis focused on MEG source modeling.

He is currently with the R\&D division of CyberLink Corp., Taipei, Taiwan.

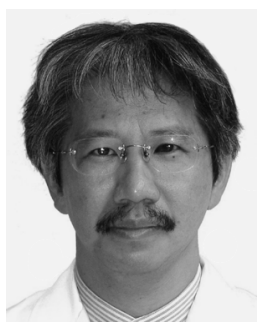

Jen-Chuen Hsieh received the M.D. degree from National Yang-Ming University, Taipei, Taiwan, in 1983. He received the Ph.D. in neuroscience from Karolinska Institute, Stockholm, Sweden, in 1995.

He was a Research Fellow in the Karolinska Institute till 1996. He has been a medical faculty of Taipei Veterans General Hospital since 1996. $\mathrm{He}$ is currently the Director of the Laboratory of Integrated Brain Research in the Department of Medical Research and Education, Taipei Veterans General Hospital. He is a Professor of Faculty of Medicine (Department of Psychiatry and Anesthesiology), Institute of Health Informatics and Decision Making, School of Medicine and Institute of Neuroscience, School of Life Science of National Yang-Ming University. His research interests encompass human brain function, cognitive neuroscience, functional magnetic resonance imaging, magnetoencephalography, electroencephalography, and positron emission tomography.

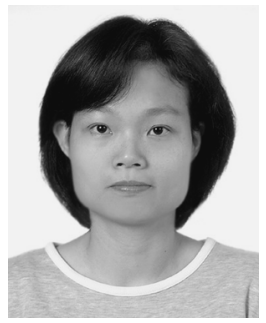

Li-Fen Chen received the B.S. and Ph.D. degrees in computer and information science from National Chiao Tung University, Hsinchu, Taiwan, in 1993 and 2000 , respectively.

She was a Postdoctoral Research Fellow with the Institute of Information Science, Academia Sinica, Taipei, Taiwan, where she was engaged in pattern recognition research from 2000 to 2001. She then joined, also as a Postdoctoral Research Fellow, the Laboratory of Integrated Brain Research, Taipei Veterans General Hospital, Taipei, Taiwan, from 2001 to 2002. She is currently an Assistant Research Fellow with the Center for Neuroscience, National Yang-Ming University, Taipei, Taiwan. Her research interests include pattern recognition, medical image analysis, and functional brain imaging through multimodal approaches combining functional magnetic resonance imaging, magnetoencephalography, and electroencephalography. 